\title{
Software Design for Dynamic Reactive Compensation System WenqiangWang ${ }^{a}, Y_{\text {YouxinYuan }}{ }^{\mathrm{b}}$, Jing Chen $^{\mathrm{c}}$, XuesongZhou $^{\mathrm{d}}$ School of Automation, Wuhan University of Technology, Wuhan, 430070, China \\ aemail:Wangwq901@qq.com, bemail: YYX2000@263.net, ${ }^{\mathrm{a}} \mathrm{email}: j i n g c h e n 680 @ 163 . c o m$, demail:86382703@qq.com
}

Keywords: nonlinear and impact loads, reactive power compensation, dynamic compensation, software design

\begin{abstract}
The nonlinear and impact loads in the power network consume large amounts of reactive power. And the amount of the reactive power compensation is different in a state of work. The traditional reactive power compensator is difficult to meet the needs of the reactive power compensation. Therefore, a method for the dynamic reactive power compensation of the composite load has been proposed by the author. Based on this method and its hardware design, the software design for the dynamic reactive power compensator is designed in this paper. The following works have been done in the study: software design requirements of the dynamical reactive power compensator, PLC program design of the dynamical reactive power compensator, monitoring system software design of the dynamical reactive power compensator. The software design is characterized by fast, dynamic and smooth reactive power compensation, friendly Human-machine Interface and bright prospect in application.
\end{abstract}

\section{Introduction}

In recent years, with the expansion of the industrial application, nonlinear and impact loads consume large amounts of reactive power. It leads to the reduction of the power factor. With the huge change of the industrial environment, the traditional reactive power compensator is difficult to meet the needs of the reactive power compensation [1]-[3].

Though, a method for the dynamic reactive power compensation of the composite load has been proposed by the author [4]. And the hardware system of the dynamic reactive power compensation has been put forward. It consists of power module, HMI, data collection module, controller PLC, I/O interface, D/A converter, pulse trigger module, AC contactor and power conversion module[5][7].

Therefore, based on this method and its hardware design, the software design for the dynamic reactive power compensator is designed in this paper. The following works have been done in the study: software design requirements of the dynamical reactive power compensator, PLC program design of the dynamical reactive power compensator, monitoring system software design of the dynamical reactive power compensator.

\section{Software Design Requirements of the Dynamical Reactive Power Compensator}

PLC.The data is initialized, collected and processed. When nonlinear and impact loads are running and consumingthe reactive power, the capacitance is switched on by PLC appropriately according to the power factor and the needed reactive power. When it is overcompensated, the inductance value is adjusted by PLC to absorb the amount of overcompensation reactive power.

Human-machine Interface (HMI).Electrical parameters, such as voltage, current, power factor, and reactive compensation state are displayedon the touch screen in real time. Sound light alarm is achieved automatically when errors happen, such as over-current and over-voltage. Measures are taken to protect the device from being damaged. 


\section{PLC Program Design of the Dynamical Reactive Power Compensator}

PLC program components for the dynamical reactive power compensator are shown in Fig.1.

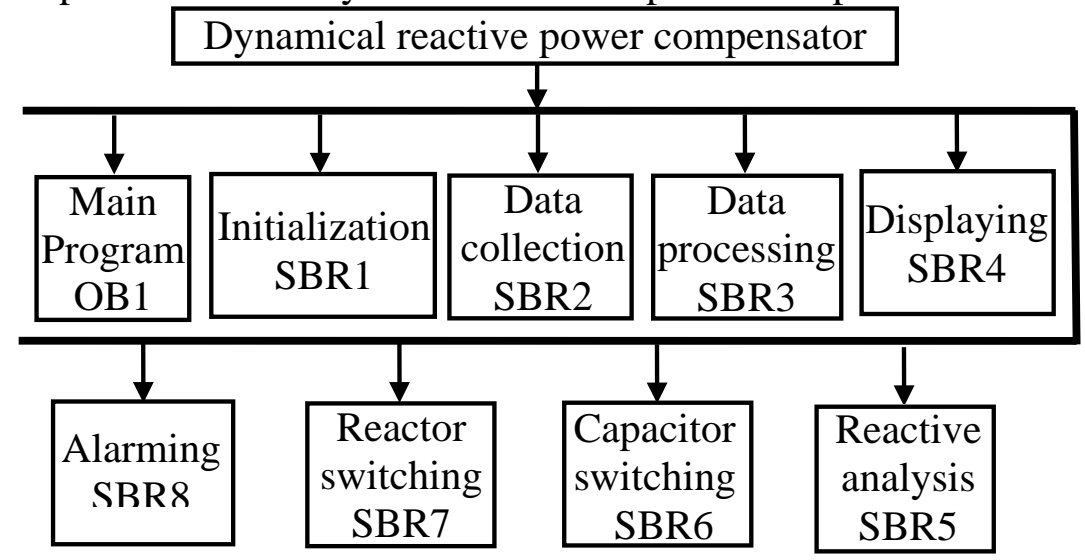

Fig.1. PLC components for the dynamical reactive power

In Fig.1, the PLC program consists of one main program and eight subprograms.

Main program (OB1). The main program is run during each scan cycle of PLC. The subprogram is called by the main program.

Initialization subprogram (SBR1). The data is initialized to the original value. Once the device start running, the data can be used immediately.

Data collection subprogram (SBR2). Electrical parameters outside the PLC controller are sampled and are sent to PLC.

Data processing subprogram (SBR3). The original data outside the PLC controller is processed to available data for PLC.

Displaying subprogram (SBR4). Electrical parameters, such as voltage, current, power factor, and reactive compensation state are displayed on the touch screen in real time.

Reactive analysis subprogram (SBR5). The amount of the demanding reactive power is calculated according to sampled electrical parameters. Then, appropriate capacitor banks can be switched on by PLC.

Capacitor switching subprogram (SBR6). Appropriate capacitor banks are switched on according to the power factor and the needed reactive power.

Reactor switching subprogram (SBR7). When it is overcompensated, the inductance value is adjusted by PLC to absorb the amount of overcompensation reactive power.

Alarming subprogram (SBR8). Sound light alarm is achieved automatically when errors happen, such as over-current and over-voltage.

Flow chart of the main program. Flow chart of the main program for PLC is shown in Fig.2. 


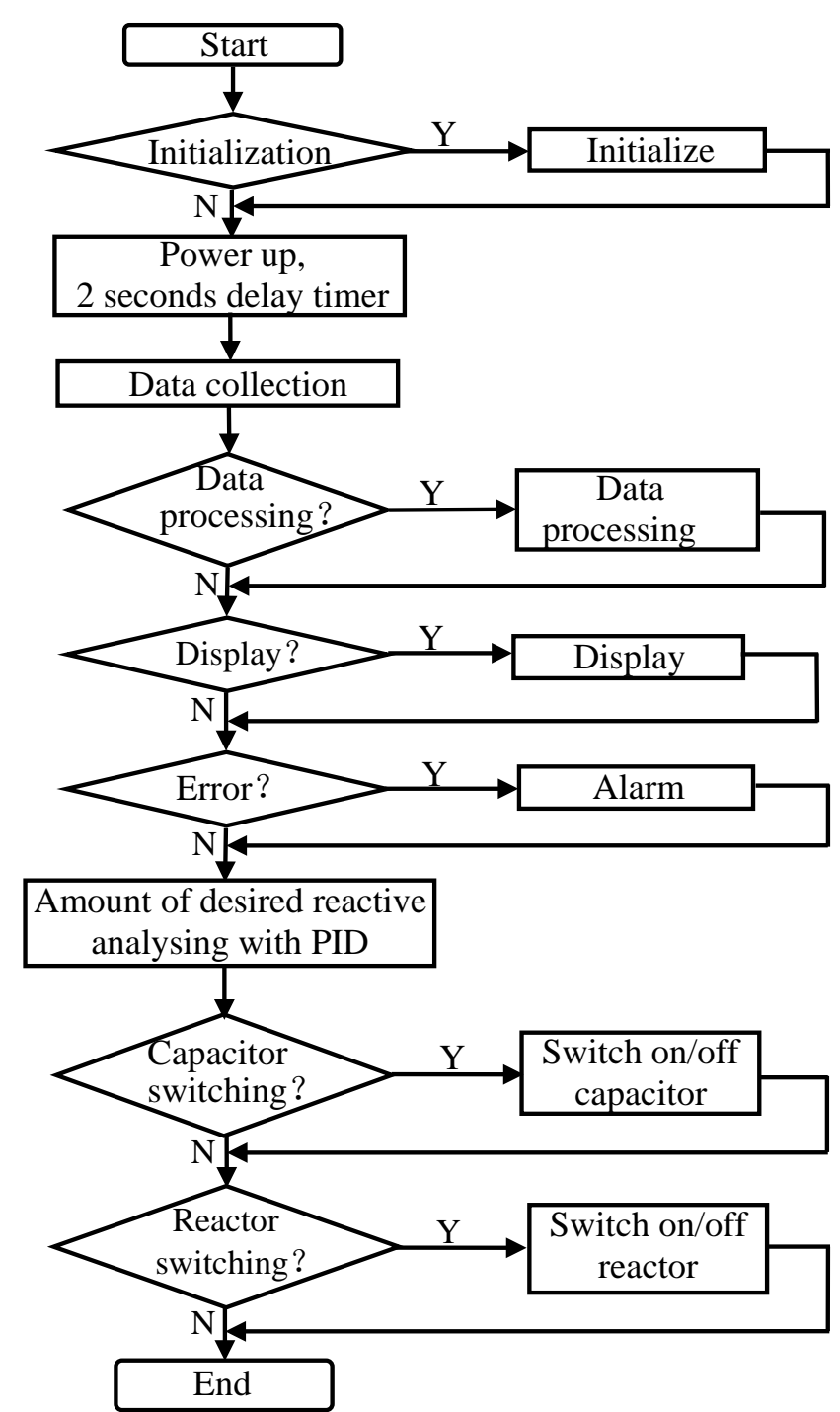

Fig.2. Flow chart of the main programfor PLC

\section{Monitoring System Software Design of the Dynamical Reactive Power Compensator}

TPC7602K is chosen as the touch screen.The monitoring interface is built via MCGS. The system state can be monitored in real time.

Main interface.Topology of the reactive compensator is on the left. Buttons of the connecting real-time monitor interface, the compensation control interface and the alarming interface are on the right. The main interface of the monitoring system is shown in Fig.3.

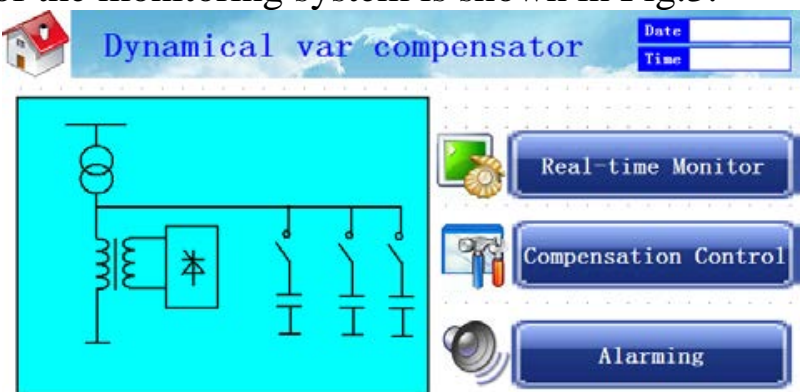

Fig.3. The main interface of the monitoring system

Real-time monitor interface.It consists of two parts. One part is real-time voltage and current display, and the other part is real-time power factor display.Because the data is processed in the data processing subprogram, the values of electrical parameters can be showed on the displaymoduledirectly. 
Compensationcontrol interface. The state of capacitor banks and its written description are showed on the touch screen. Capacitor banks can also be switched on/off manually to debug the dynamic reactive compensator. When capacitor banks are switched on, the indicator lamp is green. When capacitor banks are switched off, the indicator lamp is red.

Alarming interface. When the system breaks down and gives an alarm, the alarm information is shown with written and pictorial descriptions on the touch screen. When the error is removed, the alarm message disappears.

\section{Conclusion}

The nonlinear and impact loads in the power network consume large amounts of reactive power. And the amount of the reactive power compensation is different in a state of work. The traditional reactive power compensator is difficult to meet the needs of the reactive power compensation. Therefore, a method for the dynamic reactive power compensation of the composite load has been proposed by the author. Based on this method and its hardware design, the software design for the dynamic reactive power compensator is designed in this paper. The following works have been done in the study: software design requirements of the dynamical reactive power compensator, PLC program design of the dynamical reactive power compensator, monitoring system software design of the dynamical reactive power compensator. The research of this paper has laid an important foundation for this dynamic reactive power compensator in industrial applications.

\section{Acknowledgement}

This work was financially supported by the projects of Hubei Science and Technology Plans of China (Granted No: 2013BAA009).

\section{References}

[1]Jing Chen, Qijian Cheng, Youxin Yuan, Wei Yang.Research on the SVC Auto-Compensating Method Based on the Variable Reactor[J].Applied Mechanics and Materials, 2013321-32414141417.

[2]Youxin Yuan, Yiping Xiao,Research on Impedance Conversion Mechanism of Variable Reactor [J].Journal of Wuhan University of Technology, 200830(3)133-135.

[3]Haiping Lin, Jing Chen, Youxin Yuan, Ming Chen, Zhou Chen,Simulation Research on Passive Dynamic Harmonic Filter[J].Journal of Wuhan University of Technology, 201335(3)316-319.

[4]QiJian Cheng, Jing Chen, You Xin Yuan, Xue Song Zhou, Shi Jie Deng,Research on a Dynamic Reactive Power Compensation Method of Composite Power Load[J]. Applied Mechanics and Materials, 2014602-6052840-2843.

[5]Yifei Wang, Youxin Yuan, Jing Chen, Qijian Cheng,A Dynamic Reactive Power Compensation Method of Super High-Power and High-Voltage Motor[J].Applied Mechanics and Materials, 2014 602-6052828-2831.

[6]Yongzhong Ding, Youxin Yuan, Yiping Xiao, Xufang Zhang, Kaihua Cui,Research on Variable Reactor Based on Power Convert[J].Journal of Wuhan University of Technology, 200830(3) 136139.

[7]Youxin Yuan, Shanshan Cai, Quanju Ban, Tanfu Xiao, Tieliang Xu,Research on Topology of the Static Reactive Power Compensator Based on the Controlled Reactance[J].Journal of Wuhan University of Technology, 2009 31(12)120-122. 